A. Fujiki

Nagoya Math. J.

Vol. 85 (1982), 189-211

\title{
ON THE DOUADY SPACE OF A COMPACT COMPLEX SPACE IN THE CATEGORY $\mathscr{C}$
}

\begin{abstract}
AKIRA FUJIKI
\section{Introduction}

Let $X$ be a complex space. Let $D_{X}$ be the Douady space of compact complex subspaces of $X[6]$ and $\rho_{X}: Z_{X} \rightarrow D_{X}$ the corresponding universal family of subspaces of $X$. Thus there is a natural embedding $Z_{X} \subseteq D_{X}$ $\times X$ such that $\rho_{X}$ is induced by the projection $D_{X} \times X \rightarrow D_{X}$. Let $\pi_{X}: Z_{X}$ $\rightarrow X$ be induced by the other projection $D_{X} \times X \rightarrow X$. For any irreducible component $D_{\alpha}$ of $D_{X \text {, red }}$ we denote by $\rho_{\alpha}: Z_{\alpha} \rightarrow D_{\alpha}$ the universal family restricted to $D_{\alpha}$, and set $\pi_{\alpha}=\pi_{X \mid Z_{\alpha}}: Z_{\alpha} \rightarrow X$, where $D_{X \text {, red }}$ is the underlying reduced subspace of $D_{X}$. On the other hand, we have introduced in [9] a category $\mathscr{C}$ of compact complex spaces as follows (cf. also [10]). A compact complex space $X$ is in $\mathscr{C}$ if and only if there exist a compact Kähler manifold $Y$ and a generically surjective meromorphic map $h: Y \rightarrow$ $X_{\text {red }}, X_{\text {red }}$ being as above. Then the main purpose of this paper is to prove the following theorem: Let $X$ be a compact complex space in $\mathscr{C}$. Then for every irreducible component $D_{\alpha}$ of $D_{X \text {, red }}$ such that $Z_{\alpha}$ is reduced, $D_{\alpha}$ is compact and again belongs to $\mathscr{C}$. The proof also shows that if $X$ is Moishezon, then $D_{\alpha}$ also is Moishezon, which is a special case of a theorem of Artin [1]. Moreover since the Barlet space $B(X)$ of compact cycles of $X$ [4] is a proper holomorphic image of the union of those irreducible components of $D_{X \text {, red }}$ for which $Z_{\alpha}$ are reduced and of pure fiber dimension, the result also implies that every irreducible component of $B(X)$ is again in $\mathscr{C}$ if $X$ is in $\mathscr{C}$. Here we note that the same result as above was also obtained by Campana [5] independently.

The arrangement of this article is as follows. In $\S 1$ and $\S 2$ we define respectively the notion of a Moishezon morphism and of a morphism in the category $\mathscr{C} / S$, which is a relative version of the category $\mathscr{C}$ above, and summarize some functorial properties of these morphisms. In $\S 3$ we
\end{abstract}

Received December 21, 1979. 
make some general study on the irreducibility of general fiber of a morphism, in part to be used in $\S 5$. Then in $\S 4$ we give the main ingredient of the proof of our theorem, Proposition 4, which states that if the general fiber of $\rho_{\alpha}: Z_{\alpha} \rightarrow D_{\alpha}$ is reduced and irreducible, then $\pi_{\alpha}$ defined above is Moishezon. In fact, combining this with the results in $\S \S 1$ and 2 we obtain the theorem immediately in this special case. The reduction of the general case to this special case will then be given in $\S 5$, thus completing the proof of the theorem. Actually our theorem is expected to

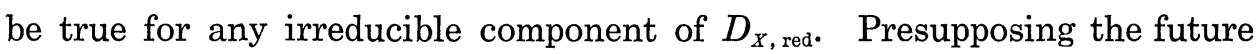
investigation of this problem along the line of [9] and in view of an application [11] also, we have developed our exposition in the relative form as in [9] so that the above theorem is also true in this generalized form (see Theorem in $\S 5$ for the precise statement). Finally in the Appendix we give a direct proof of Lemma 2 .

Notation. Let $f: X \rightarrow S$ be a morphism of complex spaces. Then for any morphism $\alpha: T \rightarrow S$ we often write $X_{T}=X \times_{S} T$ and $f_{T}: X_{T} \rightarrow T$ for the natural projection. For instance if $U \subseteq S$ is open, $f_{U}$ is the induced morphism $X_{U}=f^{-1}(U) \rightarrow U$. In particular if $T=\{s\}$ is a point of $S$ we write $X_{s}$ instead of $X_{\{s\}}$. For a complex space $X, X_{\text {red }}$ denotes the underlying reduced analytic subspace.

\section{$\S 1$. Moishezon morphisms}

(1.1) We fix notation and terminology for meromorphic maps. Let $f: X \rightarrow S$ and $g: Y \rightarrow S$ be morphisms of reduced complex spaces. Then a meromorphic $S$-map $\alpha: X \rightarrow Y$ from $X$ to $Y$ is a reduced analytic subspace $\Gamma \subseteq X \times{ }_{s} Y$ such that the natural projection $p: \Gamma \rightarrow X$ is a proper bimeromorphic morphism in the sense that $p$ is proper and that there is a dense Zariski open subset $U$ (resp. $V$ ) of $\Gamma$ (resp. $X$ ) such that $p$ induces an isomorphism of $U$ and $V$. We call $\alpha$ a (proper) bimeromorphic S-map, or being $S$-bimeromorphic, if the natural projection $q: \Gamma \rightarrow Y$ also is a proper bimeromorphic morphism. We say that $f$ and $g$ are bimeromorphic if there is a bimeromorphic $S$-map of $X$ to $Y$.

If $f$ is proper in the above definition, $q(\Gamma)$ is an analytic subspace of $Y$ and is called the image of $X$ by $\alpha$. On the other hand, $\alpha$ is called generically surjective (resp. generically finite) if $q(\Gamma)$ contains a dense Zariski open subset of $Y$ (resp. $q$ is generically finite). When $f$ is proper, the generic surjectivity is equivalent to saying that $Y=q(\Gamma)$. 
Given a meromorphic $S$-map $\alpha: X \rightarrow Y$ as above we often identify $\alpha$ with the induced $S$-morphism $\alpha^{\prime}=\left.q p^{-1}\right|_{V}: V \rightarrow Y$. Then the subspace $\Gamma$ above is recovered from $\alpha^{\prime}$ as the closure in $X \times_{s} Y$ of the graph $\Gamma_{\alpha^{\prime}} \subseteq$ $V \times{ }_{S} Y$ of $\alpha^{\prime}$ and is called the graph of $\alpha^{\prime}$. Then an $S$-morphism is nothing but the meromorphic $S$-map $\alpha$ for which we can take $V=X$.

Let $f: X \rightarrow S$ and $g_{i}: Y_{i} \rightarrow S, 1 \leqq i \leqq m$, be morphisms of complex spaces and $\alpha_{i}: X \rightarrow Y_{i}$ be meromorphic $S$-maps. Then we can define naturally a meromorphic $S$-map $\prod_{i} \alpha_{i}: X \rightarrow Y_{1} \times_{s} \cdots \times_{s} Y_{m}$ called the product of $\alpha_{i}$ over $S$; one verifies readily that the closure of the graph of the $S$-morphism $\alpha_{1}^{\prime} \times{ }_{s} \cdots \times_{s} \alpha_{m}^{\prime}$ is analytic in $X \times_{s} Y_{1} \times_{s} \cdots \times_{s} Y_{m}$ where $\alpha_{i}^{\prime}$ for $\alpha_{i}$ has the same meaning as $\alpha^{\prime}$ for $\alpha$ as above.

For later reference we recall here the analytic Chow lemma due to Hironaka [14], [15].

(1.1.1) Let $\alpha: X \rightarrow Y$ be a meromorphic $S$-map as above. Then there exist a complex manifold $X^{*}$, and a projective bimeromorphic morphism $h: X^{*} \rightarrow X$ such that the composition $\alpha h: X^{*} \rightarrow Y$ is a morphism.

(1.2) Let $f: X \rightarrow S$ be a proper morphism of complex spaces. We call $f$ locally projective if for every relatively compact open subset $Q$ of $S$ there is an invertible sheaf $\mathscr{L}=\mathscr{L}(Q)$ defined on $X$ such that $\left.\mathscr{L}\right|_{X_{Q}}$ is $f_{Q}$-ample (cf. Notation). (In this case we simply say that $\mathscr{L}$ is $f_{Q^{-}}$-ample.) Thus if $f$ is locally projective, then $f_{Q}$ is projective for every relatively compact open subset $Q \subseteq S$.

(1.2.1) A composition of two locally projective morphisms is again locally projective.

Proof. Let $f: X \rightarrow Y, g: Y \rightarrow Z$ be locally projective. Let $h=g f: X$ $\rightarrow Z$. Let $Q$ be any relatively compact open subset of $Z$. Take another relatively compact open subset $Q^{\prime}$ of $Z$ with $Q \in Q^{\prime}$. Then $\tilde{Q}^{\prime}=g^{-1}\left(Q^{\prime}\right)$ is a relatively compact open subset of $Y$. Take an invertible sheaf $\mathscr{L}$ on $X$ (resp. $\mathscr{F}$ on $Y$ ) which is $f_{\tilde{Q}^{\prime}}$-ample (resp. $g_{Q^{\prime}}$-ample). Then it is easy to see that $\mathscr{L} \otimes_{\mathscr{O}_{X}} f^{*} \mathscr{F}{ }^{m}$ is $h_{Q^{-a m p l e}}$ for all sufficiently large $m$ (cf. EGA II, 4.6.13 (ii)). Thus $h$ is locally projective.

(1.3) Let $f: X \rightarrow S$ be a locally projective morphism.

(1.3.1) If $X$ has only a finite number of irreducible components, then $f$ is $S$-bimeromorphic to a projective morphism.

Proof. Let $Q$ be any relatively compact open subset of $S$ such that 
$X_{Q}$ meets every irreducible component of $X . \quad$ Let $\mathscr{L}$ be an invertible sheaf on $X$ which is $f_{Q}$-ample. Restricting $Q$ and replacing $\mathscr{L}$ by its high power $\mathscr{L}^{n}, n \gg 0$, we may assume that $\mathscr{L}$ is even $f_{Q}$-very ample. Let $\alpha: X \rightarrow$ $\boldsymbol{P}\left(f_{*} \mathscr{L}\right)$ be the natural meromorphic $S$-map of $X$ into the projective fiber space $\boldsymbol{P}\left(f_{*} \mathscr{L}\right)$ over $S$ associated to the coherent analytic sheaf $f_{*} \mathscr{L}$ on $S$ (cf. [3], [13]). By our assumption $\alpha$ is an embedding on $X_{Q}$. Since $X_{Q}$ meets every irreducible component of $X$, this implies that $\alpha$ is bimeromorphic onto its image. Hence $f$ is bimeromorphic to a projective morphism.

Q.E.D.

From the above proof follows also the following:

(1.3.2) Let $f: X \rightarrow S$ be as in (1.3.1). Then there exist an invertible sheaf $\mathscr{L}$ on $X$ and a dense Zariski open subset $W$ of $S$ such that $\mathscr{L}$ is $f_{W^{-}}$-(very) ample.

(1.4) Definition. Let $f: X \rightarrow S$ be a proper morphism of reduced complex spaces. We call $f$ Moishezon if $f$ is bimeromorphic to a locally projective morphism $g: Y \rightarrow S$. By (1.3.1) when $X$ has only a finite number of irreducible components, $f$ is Moishezon if and only if $f$ is bimeromorphic to a projective morphism.

Remark. In [17] Moishezon introduced the notion of an $A$-space over another complex space, and stated some of their fundamental properties. From his definition it follows readily that for a proper morphism $f: X \rightarrow$ $S$ of reduced complex spaces $X$ is an $A$-space over $S$ if and only if $f$ is locally Moishezon in the sense that for each point $s \in S$ there is a neighborhood $s \in U$ such that the induced morphism $f_{U}: X_{U} \rightarrow U$ is Moishezon in the sense defined above.

(1.5) Clearly the Moishezon property of a morphism is invariant under $S$-bimeromorphic equivalence. We now list some fundamental properties of Moishezon morphisms.

1) A composition of two Moishezon morphisms are again Moishezon.

2) $f: X \rightarrow S$ is Moishezon if and only if for each irreducible component $X_{i}$ of $X$ the restriction $f=\left.f\right|_{X_{i}}: X_{i} \rightarrow S$ is Moishezon.

3) If $f$ is Moishezon, there are a locally projective morphism $g: X^{*}$ $\rightarrow S$ with $X^{*}$ nonsingular and a bimeromorphic $S$-morphism $h: X^{*} \rightarrow X$.

4) Suppose that there exist a locally projective morphism $g: Y \rightarrow S$ and a generically finite meromorphic $S$-map $h: X \rightarrow Y$. Then $f$ is Moishezon.

Proof. 1) and 3) follows from (1.1.1) and (1.2.1). Let $\mu: \tilde{X} \rightarrow X$ be the 
normalization of $X$. Since $\mu$ is bimeromorphic, $f$ is Moishezon if and only if $f \mu$ is Moishezon. From this 2) follows readily. 4) Changing $f$ under bimeromorphic equivalence we may assume that $h$ is a morphism. Let $h=h_{2} h_{1}$ with $h_{1}: X \rightarrow X^{*}$ and $h_{2}: X^{*} \rightarrow Y$ be the Stein factorization of $h$, where $h_{1}$ is a bimeromorphic, and $h_{2}$ is a finite, $S$-morphisms. Since a finite morphism is projective, $g h_{2}: X^{*} \rightarrow S$ is locally projective by (1.2.1), and hence 4).

(1.6) Less trivial to prove is the following:

Proposition 1. Let $f: X \rightarrow S$ be a Moishezon morphism, and $g: Y \rightarrow$ $S$ a proper morphism, of reduced complex spaces. Suppose that there is a generically surjective meromorphic S-map $h: X \rightarrow Y$. Then $g$ also is Moishezon.

Proof. By (1.5) 2) we may assume that $Y$, and then $X$ and $S$ also, are irreducible. By (1.1.1) and (1.5) 3) we may further assume that $f$ is locally projective, $X$ is nonsingular and $h$ is a morphism. Then there is a dense Zariski open subset $V_{0}$ of $Y$ such that $V_{0}$ is nonsingular and $h_{V_{0}}: X_{V_{0}} \rightarrow V_{0}$ is smooth. Let $\mathscr{L}$ be an invertible sheaf on $X$ which is $f_{W^{-}}$ ample for some dense Zariski open subset $W$ of $S$ (1.3.2). Restricting $V_{0}$ we may assume that $V_{0} \subseteq Y_{W}$. Then if $n$ is sufficiently large, say, $n \geqq n_{0}$ for some $n_{0}>0$, there is a dense Zariski open subset $V_{n}$ of $Y$ such that $V_{n} \subseteq V_{0}$ and $H^{1}\left(X_{y}, \mathscr{L}_{y}^{n}\right)=0$ for all $y \in V_{n}$ where $\mathscr{L}_{y}^{n}=\mathscr{L}^{n} \otimes_{O_{X}} \mathcal{O}_{X y}$. Let $\mathscr{E}_{n}=h_{*} \mathscr{L}^{n}$. Then $\mathscr{E}_{n}$ is a coherent analytic sheaf on $Y$ which is locally free of rank, say $r_{n}$, on $V_{n}$ (cf. [3, p. 122, Cor. 3.9]). Moreover taking $n_{0}$ larger if necessary we may assume that $r_{n}>0$ for $n \geqq n_{0}$. On the other hand, by [20] we can find a proper surjective bimeromorphic morphism $\sigma_{n}: \tilde{Y}_{n} \rightarrow Y$ such that $\tilde{\mathscr{E}}_{n}=\sigma_{n}^{*} \mathscr{E} \mid \mathscr{T}_{n}, \mathscr{T}_{n}$ being the torsion part of $\sigma_{n}^{*} \mathscr{E}$, is locally free of rank $r_{n}$ on $\tilde{Y}_{n}$. Moreover we can assume that $\sigma_{n}$ gives an isomorphism of $\tilde{V}_{n}=\sigma_{n}^{-1}\left(V_{n}\right)$ onto $V_{n}$. Let $\tilde{g}_{n}=\sigma_{n} g: \tilde{Y}_{n} \rightarrow S$ and set $\mathscr{M}_{n}=\wedge^{r_{n} \tilde{E}_{n}}$, where $\bigwedge^{r_{n}}$ denotes the $r_{n}$-th exterior product. Then $\mathscr{M}_{n}$ is an invertible sheaf on $\tilde{Y}_{n}$. Let $\alpha_{n}: \tilde{Y}_{n} \rightarrow \boldsymbol{P}\left(\tilde{g}_{n *} \mathscr{M}_{n}\right)$ be the natural meromorphic $S$-map from $\tilde{Y}_{n}$ to the projective fiber space $\boldsymbol{P}\left(\tilde{g}_{n *} \mathscr{M}_{n}\right)$ over $S$ associated to the coherent analytic sheaf $\tilde{g}_{n *} \mathscr{M}_{n}$ on $S$ (cf. [3, IV, § 1]). Then we show that for a sufficiently large $n, \alpha_{n}$ is generically finite; then by (1.5) d) the proposition would follow.

For this purpose it is enough to show that for some $n \geqq n_{0}$, for some $\tilde{y} \in \tilde{Y}_{n}$ and for some neighborhood $U$ of $s=\tilde{g}_{n}(y)$ in $S$, the following holds 
true; there are sections $\varphi_{1}, \cdots, \varphi_{d} \in \Gamma\left(\tilde{Y}_{n, U}, \mathscr{M}_{n}\right)$ such that the meromorphic $U$-map $\Phi: \tilde{Y}_{n, U} \rightarrow U \times \boldsymbol{C P}^{d-1}$ associated to $\varphi_{\alpha}$ is holomorphic and locally biholomorphic at $\tilde{y}$, where $\tilde{Y}_{n}$ is over $S$ by $\tilde{g}_{n}$. (Note that $\tilde{Y}_{n}$ is irreducible.) First we take $n, \tilde{y}$ and $U$ in such a way that $\tilde{y} \in \tilde{V}_{n}, U$ is a sufficiently small Stein open neighborhood of $s$, and that $H^{1}\left(X_{U}, m_{y}^{2} \mathscr{L}^{n}\right)=0$, where $y=\sigma_{n}(\tilde{y})$ and $m_{y}$ is the maximal ideal of $\mathcal{O}_{Y}$ at $y$. Clearly this is possible since $\mathscr{L}$ is $f_{W}$-ample and $y \in V_{n} \subseteq Y_{W}$. Then in particular the restriction map $\beta_{n}: \Gamma\left(X_{U}, \mathscr{L}^{n}\right) \rightarrow \Gamma\left(X_{U}, \mathscr{L}_{(2)}^{n}\right)$ is surjective, as follows from the long exact sequence associated to the short one

$$
0 \longrightarrow m_{y}^{2} \mathscr{L}^{n} \longrightarrow \mathscr{L}^{n} \longrightarrow \mathscr{L}_{(2)}^{n} \longrightarrow 0
$$

where we have put $\mathscr{L}_{(2)}^{n}=\mathscr{L}^{n} \otimes_{\mathcal{O}_{X}} \mathcal{O}_{X} / m_{y}^{2} \mathcal{O}_{X}$. Further since

$$
H^{1}\left(X_{U}, m_{y} \mathscr{L}^{n} / m_{y}^{2} \mathscr{L}^{n}\right) \cong H^{1}\left(X_{U}, \mathscr{L}^{n} \otimes_{o_{X}} m_{y} / m_{y}^{2}\right) \cong H^{1}\left(X_{y}, \mathscr{L}_{y}^{n}\right) \otimes_{c} m_{y} / m_{y}^{2}=0,
$$

from the short exact sequence $0 \rightarrow m_{y} \mathscr{L}^{n} / m_{y}^{2} \mathscr{L}^{n} \rightarrow \mathscr{L}_{(2)}^{n} \rightarrow \mathscr{L}_{y}^{n} \rightarrow 0$ we have the exact sequence

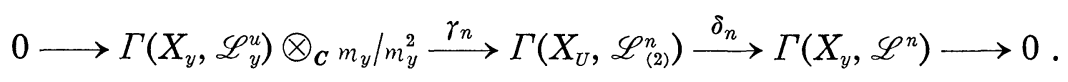

Fix $n$ and write $r=r_{n}$. Then take and fix a base $\left(\bar{\psi}_{1}^{0}, \cdots, \bar{\psi}_{r}^{0}\right)$ of $\Gamma\left(X_{y}, \mathscr{L}_{y}^{n}\right)$. Let $\left(y_{1}, \cdots, y_{m}\right), m=\operatorname{dim} Y$, be a local coordinate system around $y$ of $Y$ and $\bar{y}_{i}$ the residue classes of $y_{i}$ in $\mathcal{O}_{Y} / m_{y}^{2} \mathcal{O}_{Y}$. Then we take any base $\left(\bar{\psi}_{1}, \cdots, \bar{\psi}_{d}\right), d=r(m+1)$, of $\Gamma\left(X_{U}, \mathscr{L}_{(2)}^{n}\right)$ satisfying the following conditions; $\delta_{n}\left(\bar{\psi}_{i}\right)=\bar{\psi}_{i}^{0}, 1 \leqq i \leqq r$, and $\bar{\psi}_{k r+j}=\gamma_{n}\left(\bar{y}_{k} \bar{\psi}_{j}\right), 1 \leqq k \leqq m, 1 \leqq j \leqq r$, where $\bar{y}_{k} \bar{\psi}_{j}=\bar{\psi}_{j} \otimes \bar{y}_{k} \in \Gamma\left(X_{y}, \mathscr{L}_{y}\right) \otimes_{c} m_{y} / m_{y}^{2}$. For each $1 \leqq k \leqq d$ take and fix $\psi_{k}$ $\in \Gamma\left(X_{U}, \mathscr{L}^{n}\right)$ with $\beta_{n}\left(\psi_{k}\right)=\bar{\psi}_{k}$. With respect to the natural identification $\Gamma\left(X_{U}, \mathscr{L}^{n}\right) \cong \Gamma\left(Y_{U}, \mathscr{E}_{n}\right) \subseteq \Gamma\left(\tilde{Y}_{n, U}, \widetilde{\mathscr{E}}_{n}\right)$, we consider $\psi_{i}$ naturally as sections of $\widetilde{\mathscr{E}}_{n}$ on $\tilde{Y}_{n, U}$. Then for any $1 \leqq i_{1} \ldots \leqq i_{r} \leqq d$ define $\varphi_{i_{1} \cdots i_{r}} \in \Gamma\left(\tilde{Y}_{n, U}, \mathscr{M}_{n}\right)$ by $\varphi_{i_{1} \cdots i_{r}}=\psi_{i_{1}} \wedge \cdots \wedge \psi_{i_{r}}$. We claim that these $\varphi_{\alpha}=\varphi_{i_{1} \cdots i_{r}}$ have the desired properties.

Since the problem is local around $y$ and $\sigma_{n}$ gives a natural isomorphism of $\tilde{V}_{n}$ and $V_{n}$, in what follows we identify $\tilde{V}_{n}$ and $V_{n}$ by $\sigma_{n}$ and therefore $\tilde{y}$ with $y$ and $\left.\widetilde{\mathscr{E}}_{n}\right|_{\tilde{V}_{n}}$ with $\left.\mathscr{E}_{n}\right|_{V n}$. Further we consider $\left.\mathscr{M}_{n}\right|_{\tilde{v}_{n}}$ as an invertible sheaf on $V_{n}$ and $\psi_{i}$ as sections of $\mathscr{E}_{n}$ on $Y_{U} \cap V_{n}$. Now $\psi_{1}, \cdots, \psi_{r}$ define a trivialization $\mathscr{E}_{n} \cong \mathcal{O}_{Y}^{r}$ of $\mathscr{E}_{n}$, and hence also $\mathscr{M}_{n} \cong \mathcal{O}_{Y}$ of $\mathscr{M}_{n}$, in some neighborhood $N$ of $y$. In particular we may consider each $\psi_{i}\left(\right.$ resp. $\varphi_{i_{1} \ldots i_{r}}$ ) as an $r$-tuple of holomorphic functions (resp. a holomorphic function) on $N$. Then we have by construction $\psi_{i}=(0, \cdots, 0,1,0, \cdots, 0)$ 
for $1 \leqq i \leqq r$ where 1 is on the $i$-th place and $\psi_{k r+j} \equiv\left(0, \cdots, 0, y_{k}, 0, \cdots, 0\right)$ modulo $m_{y}^{2}$, where $y_{k}$ is on the $j$-th place. Hence we have $\varphi_{1 \ldots r}(y)=\psi_{1}$ $\wedge \cdots \wedge \psi_{r}(0) \neq 0$ and $\varphi_{1 \cdots \hat{k} \cdots r(k r+k)}=\psi_{1} \wedge \cdots \wedge \hat{\psi}_{k} \wedge \cdots \wedge \psi_{r} \wedge \psi_{k r+k} \equiv$ $y_{k}$ modulo $m_{y}^{2}$ where $\hat{u}$ implies the absense of $u$. The former implies that $\Phi$ is holomorphic at $y$ and the latter implies that $\Phi$ is locally biholomorphic at $y$. Hence our claim is verified.

Q.E.D.

(1.7) Let $f: X \rightarrow S$ be a Moishezon morphism. Then:

5) For every reduced analytic subspace $X^{\prime} \subseteq X$ the induced morphism $f^{\prime}=\left.f\right|_{X^{\prime}}: X^{\prime} \rightarrow S$ is Moishezon.

6) Let $\mu: \widetilde{S} \rightarrow S$ be a morphism of reduced complex spaces. Then the induced map $f_{\tilde{S}, \text { red }}: X_{\tilde{S} \text {, red }} \rightarrow \tilde{S}$ is Moishezon.

7) Let $g: Y \rightarrow S$ be another Moishezon morphism. Then $f \times_{s} g: X$ $\times_{S} Y \rightarrow S$ also is Moishezon.

Proof. Let $g: X^{*} \rightarrow S$ and $h: X^{*} \rightarrow X$ be as in (1.5) 3). Let $Z=$ $h^{-1}\left(X^{\prime}\right)$ with reduced structure. Then $\left.g\right|_{Z}: Z \rightarrow S$ is locally projective and $\left.h\right|_{Z}: Z \rightarrow X^{\prime}$ is surjective. Hence by the above proposition $f$ is Moishezon. This proves 5). We show 6). Let $g$ and $h$ be as above. Then $h$ induces a surjective morphism $h_{\tilde{S} \text {, red }}: X_{\tilde{S} \text {, red }}^{*} \rightarrow X_{\tilde{S} \text {, red }}$ over $S$. Since $g_{\tilde{S} \text {, red }}: X_{\tilde{S} \text {, red }}^{*} \rightarrow$ $\tilde{S}$ is locally projective, 6) also follows from the above proposition. Since $f \times{ }_{s} g$ is the composition of the natural projection $X \times{ }_{s} Y \rightarrow Y$ and $g$, 7) follows from (1.5) 1) and 6) above.

\section{§2. Morphisms in $\mathscr{C} / S$}

(2.1) Definition. Let $g: Y \rightarrow S$ be a proper morphism of complex spaces. Then: 1) ([9, Def. 4.1]) $g$ is called Kähler if there exist an open covering $\left\{U_{\alpha}\right\}$ of $Y$ and a $C^{\infty}$ function $p_{\alpha}$ defined on each $U_{\alpha}$ such that for each $\alpha, p_{\alpha}$ is strictly plurisubharmonic when restricted to each fiber of $\left.g\right|_{U_{\alpha}}: U_{\alpha} \rightarrow S$ and that $p_{\alpha}-p_{\beta}$ is pluriharmonic on each $U_{\alpha} \cap U_{\beta}$. 2) $g$ is called locally Kähler if for every relatively compact open subset $Q$ of $S$ there exist $\left\{U_{\alpha}\right\}$ and $\left\{p_{\alpha}\right\}$ satisfying the condition as above except that $p_{\alpha}$ is assumed to be strictly plurisubharmonic only when restricted to each fiber of $\left.g\right|_{U_{\alpha} \cap g^{-1}(Q)}: U_{\alpha} \cap g^{-1}(Q) \rightarrow Q$.

In the above definition the real closed $(1,1)$-form $\omega_{\alpha}=\sqrt{-1} \partial \bar{\partial} p_{\alpha}$, each defined on $U_{\alpha}$, patch together to give a global real closed $(1,1)$-form $\omega$ on $Y$, which we call a relative Kähler form for $g$ (resp. for $g$ over $Q$ ).

(2.2) In the following all the morphisms considered are proper. 
1) Every (locally) projective morphism is (locally) Kähler.

2) Let $g: Y \rightarrow S$ be a (locally) Kähler morphism and $\alpha: \widetilde{S} \rightarrow S$ a morphism of complex spaces. Then the induced morphism $g_{\tilde{S}}: Y_{\tilde{S}} \rightarrow \tilde{S}$ is (locally) Kähler.

3) Let $f: X \rightarrow Y$ and $g: Y \rightarrow S$ be locally Kähler morphism of complex spaces. Then the composition $g f: X \rightarrow S$ is again locally Kähler. Conversely if $g f$ is (locally) Kähler, then $f$ also is (locally) Kähler.

4) Let $f: X \rightarrow S$ and $g: Y \rightarrow S$ be locally Kähler morphisms. Then $f \times{ }_{S} g: X \times_{S} Y \rightarrow S$ is locally Kähler.

Proof. See [9. Lemma 4.4] for 1). We show the former half of 3). Let $Q \subset Q^{\prime} \subset S$ and $\tilde{Q}=g^{-1}\left(Q^{\prime}\right)$ be as in the proof of (1.2.1) (with $Z$ replaced by $S$ ). Let $\omega_{Q^{\prime}}$ (resp. $\omega_{\tilde{Q}}$ ) be a relative Kähler form for $g$ over $Q^{\prime}$ (resp. $f$ over $\tilde{Q}$ ). Then for all sufficiently large $n>0, \omega_{\tilde{Q}}+\left.n f^{*} \omega_{Q^{\prime}}\right|_{(g f)^{-1}(Q)}$ gives a relative Kähler form for the morphism gf over $Q$ (cf. the proof of [9, Lemma 4.4]). Hence $g f$ is locally Kähler. Since $f \times_{s} g$ is a composite of the natural projection $X \times_{s} Y \rightarrow Y$ and $\left.g, 4\right)$ follows from this and 2). The other assertions follow immediately from the definition.

(2.3) Definition. Let $S$ be a reduced complex space. Then we define the category $\mathscr{C} / S$ as follows: An object of $\mathscr{C} / S$ is a proper morphism $f: X \rightarrow S$ of reduced complex spaces for which there exist a proper and locally Kähler morphism $g: Y \rightarrow S$ and a generically surjective meromorphic $S$-map $h: Y \rightarrow X$ (Notation: $f \in \mathscr{C} \mid S$ ); and a morphism in $\mathscr{C} / S$ is a morphism $u: X_{1} \rightarrow X_{2}$ of complex spaces with $f_{2} u=f_{1}$ where $f_{i}: X_{i} \rightarrow S \in \mathscr{C} \mid S, i=1,2$.

Remark. 1) Note the deviation from the notation adopted in $[9, \mathrm{p}$. 51]; there we used the notation $\mathscr{C} / S$ for the category loc- $\mathscr{C} / S$ which is defined as follows: An object of loc- $\mathscr{C} \mid S$ is a proper morphism $f: X \rightarrow S$ of complex spaces for which there exists an open covering $\left\{U_{\alpha}\right\}$ of $S$ such that $f_{U_{\alpha}}: X_{U_{\alpha}} \rightarrow U_{\alpha} \in \mathscr{C} / U_{\alpha}$ for each $\alpha$, with morphisms defined as above. 2) When $S$ is a point, we write $\mathscr{C}$ instead of $\mathscr{C} / S$. In this case the definition coincides with that given in $[9,4.3]$ except that we consider only reduced spaces here.

(2.4) We shall give some functorial properties of morphisms in $\mathscr{C} / S$ analogous to Moishezon morphisms.

1) Every Moishezon morphism belongs to $\mathscr{C} / S$.

Let $f: X \rightarrow S$ and $g: Y \rightarrow S$ be proper morphisms of reduced complex spaces. Suppose that $g \in \mathscr{C} / S$. Then: 
2) $f \in \mathscr{C} / S$ if and only if there exist a proper and locally Kähler morphism $g^{*}: Y^{*} \rightarrow S$ with $Y^{*}$ nonsingular, and a surjective $S$-morphism $h^{*}: Y^{*} \rightarrow S$.

3) For every analytic subspace $Y^{\prime}$ of $Y$ the induced morphism $\left.g\right|_{Y^{\prime}}: Y^{\prime}$ $\rightarrow S$ is again in $\mathscr{C} / S$.

4) Suppose that there is a generically surjective meromorphic $S$-map $h: Y \rightarrow X$. Then $f \in \mathscr{C} \mid S$.

5) Suppose that there is an $S$-morphism $h: X \rightarrow Y$ with $h \in \mathscr{C} / Y$. Then $f \in \mathscr{C} / S$.

6) For any reduced complex space $\tilde{S}$ over $S$ the induced morphism $g: Y_{\tilde{S}, \text { red }} \rightarrow \tilde{S}$ is in $\mathscr{C} / \tilde{S}$.

7) Suppose that $f \in \mathscr{C} / S$. Then $f \times{ }_{s} g: X \times_{S} Y \rightarrow S$ is again in $\mathscr{C} / S$.

Proof. 1) follows from (2.2) 1) and the definition of a Moishezon morphism. The proofs of 2), 3) and 4) are the same as those of 1), 2) and 3) of [9, Lemma 4.6] respectively, using (2.2) instead of [9, Lemma 4.4], and will be omitted.

5) By assumption and by 2) there exist a locally Kähler morphism $\tilde{g}: \tilde{Y} \rightarrow S$ (resp. $\tilde{h}: \tilde{X} \rightarrow Y$ ) and a surjective $S$-(resp. $X$-)morphism $\alpha: \tilde{Y} \rightarrow Y$ (resp. $\beta: \tilde{X} \rightarrow X$ ). Then the natural map $\gamma: \tilde{X} \times_{Y} \tilde{Y} \rightarrow S$ is locally Kähler by (2.2) 4). Moreover there is a natural surjective $S$-morphism $\tilde{X} \times_{Y} \tilde{Y} \rightarrow$ $X$, which proves 5). Let $\tilde{g}: \tilde{Y} \rightarrow S$ and $\alpha: \tilde{Y} \rightarrow Y$ be as above. Then $\tilde{Y}_{\tilde{S}}$ $\rightarrow \tilde{S}$ is locally Kähler by (2.2) 2) and there is a natural surjective $\tilde{S}$ morphism $\tilde{Y}_{\tilde{S}} \rightarrow Y_{\tilde{S}}$. This proves 6). 7) then follows from 5) and 6) as in the proof of (2.2) 4).

\section{§3. Irreducibility of the general fiber of a morphism}

(3.1) Let $f: X \rightarrow Y$ be a finite surjective morphism of reduced complex spaces. Then we call $f$ a finite (ramified) covering if each irreducible component of $X$ is mapped surjectively onto some irreducible component of $Y$.

LEMMA 1. Let $\beta: X \rightarrow Y$ be a finite covering of reduced complex spaces with $Y$ irreducible. Then there are a normal complex space $\tilde{X}$ and a finite covering $\gamma: \tilde{X} \rightarrow Y$ such that the induced morphism $\beta_{\tilde{X}}^{*}:\left(X \times_{Y} \tilde{X}\right)^{*} \rightarrow \tilde{X}$ is biholomorphic to the natural projection $E \times \tilde{X} \rightarrow \tilde{X}$, where $\left(X \times_{Y} \tilde{X}\right)^{*}$ is the normalization of $X \times_{Y} \tilde{X}$, and $E$ is a finite set considered as a 0 dimensional reduced complex space. 
Proof. Replacing $X$ and $Y$ by their normalizations $X^{\prime}$ and $Y^{\prime}$ respectively, and then considering separately the finite coverings $\beta_{i}: X_{i} \rightarrow Y^{\prime}$ induced by $\beta$ between the irreducible components $X_{i}^{\prime}$ of $X^{\prime}$ and $Y^{\prime}$, we infer readily that we may assume that both $X$ and $Y$ are normal and irreducible. Then by the argument in [21, p. 62] we can find a normal complex space $\tilde{X}$, a finite group $G$ of biholomorphic automorphisms of $\tilde{X}$ and a subgroup $H$ of $G$ such that we have the natural isomorphisms $h: X \cong \tilde{X} / H$ and $g: Y \cong \tilde{X} / G$ with $\beta=g^{-1} \pi h$, where $\tilde{X} / H$ and $\tilde{X} / G$ are the quotients of $\tilde{X}$ by $H$ and $G$ respectively endowed with their natural structures of normal complex spaces, and $\pi: X / H \rightarrow X / G$ is the natural projection. Then identifying $\pi$ with $\beta$ by the above isomorphisms, this implies the lemma as follows. Let $\Delta$ be the diagonal of $\tilde{X} \times_{\tilde{X} / G} \tilde{X}$ and let $G$ act on $\tilde{X} \times_{\tilde{X} / G} \tilde{X}$ by $\left(x_{1}, x_{2}\right) \rightarrow\left(g x_{1}, x_{2}\right)$ for each $g \in G$. Then $\tilde{X} \times_{\tilde{X} / G} \tilde{X}=$ $\bigcup_{g \in G} g \Delta$ so that $\left(\tilde{X} \times_{\tilde{X} / G} \tilde{X}\right)^{*} \cong \bigsqcup_{g \in G} g \Delta$ and each $g \Delta$ is mapped isomorphically onto $\tilde{X}$ by the second projection. Accordingly, we have $\left(\tilde{X} / H \times_{\tilde{X} / G} \tilde{X}\right)^{*} \cong \coprod_{g \in E}\left(\hat{\pi} \times \operatorname{id}_{X}\right)(g \Delta) \cong \bigsqcup_{g \in E} g \Delta \cong E \times \tilde{X}$ where $\hat{\pi}: \tilde{X} \rightarrow \tilde{X} / H$ is the natural projection and $E$ is any complete set of representatives of $G / H$ in $G$.

Q.E.D.

(3.2) Let $f: X \rightarrow S$ be a proper surjective morphism of reduced complex spaces. In what follows the 'general' fiber of $f$ is always considered with respect to the Zariski topology of $S$. For example 'the general fiber of $f$ is reduced and irreducible' means that $X_{s}$ is reduced and irreducible for every $s \in U$ for some dense Zariski open subset $U$ of $S$.

Proposition 2. Let $f: X \rightarrow S$ be as above. Then there exist a finite surjective morphism $\beta: \tilde{S} \rightarrow S$ with $\tilde{S}$ reduced, and a reduced analytic subspace $\tilde{X}$ of $X \times{ }_{S} \tilde{S}$ such that if $f: \tilde{X} \rightarrow \tilde{S}$ and $\alpha: \tilde{X} \rightarrow X$ are the naturally induced morphisms, then 1) the irreducible components of $\tilde{X}$ are mutually disjoint, 2) $\alpha$ is bimeromorphic, and in particular every irreducible component of $\tilde{X}$ is mapped bimeromorphically onto an irreducible component of $X$ and 3) the general fiber of $\tilde{f}$ is reduced and irreducible. Moreover if $f$ is flat, then we can take $\beta$ to be a finite covering.

Proof. Let $\nu: X^{\prime} \rightarrow X$ be the normalization of $X$ and let $f_{\nu}=\beta g$ with $g: X^{\prime} \rightarrow \tilde{S}$ and $\beta: \tilde{S} \rightarrow S$ be the Stein factorization of $f_{\nu}: X^{\prime} \rightarrow S$. Then we set $\tilde{X}=(\nu \times g)\left(X^{\prime}\right) \leqq X \times{ }_{S} \tilde{S}$, and define $\alpha$ and $\tilde{f}$ as above. Then clearly $\beta$ is finite surjective and 2) is satisfied. We shall show 1). Suppose that $\tilde{X}_{i} \cap \tilde{X}_{j} \neq \emptyset$ for some distinct irreducible components $\tilde{X}_{i}$ and $\tilde{X}_{j}$ of $\tilde{X}$. 
Let $\tilde{x} \in \tilde{X}_{i} \cap \tilde{X}_{j}$ be any point and $\tilde{s}=g(\tilde{x})$. Then if $X_{i}^{\prime}$ and $X_{j}^{\prime}$ are the irreducible components of $X^{\prime}$ with $(\nu \times g)\left(X_{i}^{\prime}\right)=\tilde{X}_{i}$ and $(\nu \times g)\left(X_{j}^{\prime}\right)=\tilde{X}_{j}$ respectively, then we have $X_{i, \tilde{s}}^{\prime} \neq \emptyset$ and $X_{j, \tilde{s}}^{\prime} \neq \emptyset$. Since $X_{\tilde{s}}^{\prime}$ is connected by the definition of Stein factorization, this implies that there is some irreducible component $X_{k}^{\prime} \neq X_{i}^{\prime}$ of $X^{\prime}$ such that $X_{i}^{\prime} \cap X_{k}^{\prime} \neq \emptyset$. This is a contradiction since $X^{\prime}$ is normal. Hence 1) is proved. Then the reducedness of the general fiber of $\tilde{f}$ follows from [9, Lemma 1.5]. So it remains to show that the general fiber of $\tilde{f}$ is irreducible. This in turn follows from that of $g$, and the latter can be seen as follows. Let $r: X^{*}$ $\rightarrow X^{\prime}$ be a resolution of $X^{\prime}$ and $g^{*}=g r: X^{*} \rightarrow S$. Then there is a dense Zariski open subset $V$ of $S$ such that $g_{V}^{*}: X_{V}^{*} \rightarrow V$ is smooth, and hence irreducible since each fiber of $g^{*}$ is connected as well as that of $g, X^{\prime}$ being normal. Hence $X_{\tilde{s}}^{\prime}=r\left(X_{\tilde{s}}^{*}\right)$ are also irreducible for all $\tilde{s} \in V$. Q.E.D.

Remark. In the above proof, to show the irreducibility of the general fiber of $g$, instead of resolution we can also use the fact that if $h: X \rightarrow S$ is a proper morphism with $X$ normal, then the set $\left\{s \in S ; X_{s}\right.$ is normal and $f$ is flat at each point of $\left.X_{s}\right\}$ is dense and Zariski open in $S$, which can be shown as in [9, Lemmas 1.4, 1.5] starting from a result of [2].

(3.3) We shall show that a general fiber of a proper flat morphism is irreducible if at least one fiber is reduced and irreducible. Though the result is not absolutely necessary for the proof of Theorem, it provides us with a useful criterion for the applicability of Proposition 4 in $\S 4$. First we need some lemmas.

Lemma 2. Let $f: X \rightarrow Y$ be a proper morphism of complex spaces and $y \in Y$. Then $f$ is flat at each point of $X$ if and only if for any morphism $h: D \rightarrow Y$ with $h(0)=y$, the induced morphism $f_{D}: X_{D} \rightarrow D$ is flat at each point of $X_{D, 0}$ where $D=\{t \in C ;|t|<1\}$ is the unit disc and $0 \in D$ is the origin.

Proof. This is an immediate consequence of the existence of 'platificateur' in [16, Th. 1'] (cf. also [14, Th. 2.4]). We shall also give a direct proof of the lemma in the Appendix.

CoRollary. Let $f: X \rightarrow Y$ be a proper surjective morphism of reduced and irreducible complex spaces. Let $y \in Y$. Suppose that $X_{y}$ is reduced and irreducible, and that $\operatorname{dim} X_{y}=\operatorname{dim} X-\operatorname{dim} Y$. Then $f$ is flat at every point of $X_{y}$. 
Proof. It suffices to show that for any $h: D \rightarrow Y$ with $h(0)=y, f_{D}: X_{D}$ $\rightarrow D$ is flat along $X_{D, 0}$. Since $X_{D, 0} \cong X_{y}$ is reduced and irreducible, by Nakayama we may assume that $X_{D}$ is reduced. Let $X_{D i}, 1 \leqq i \leqq m$, be the irreducible components of $X_{D}$. Restricting $D$ smaller we may further assume that $f_{D}\left(X_{D i}\right)=D$ or $\{0\}$ for each $i$. Since $X_{D, 0}$ is reduced and irreducible, if $f_{D}\left(X_{D i}\right)=\{0\}$ for some $i$, we must have $X_{D i}=X_{D, 0}$, and $i$ is unique, say $i=m$. Note that since $f_{D}$ is surjective, $m>1$. Then for $1 \leqq i<m$ we have $\operatorname{dim} X_{D i, t} \leqq \operatorname{dim} X_{D i, 0}<\operatorname{dim} X_{D, 0}$, and hence $\operatorname{dim} X_{D, t}<$ $\operatorname{dim} X_{D, 0}$, or $\operatorname{dim} X_{h(t)} \leqq \operatorname{dim} X_{y}$, for $t \neq 0$. On the other hand, our dimensional assumption implies that $\operatorname{dim} X_{y}=\operatorname{dim} X_{y^{\prime}}$ for all $y^{\prime}$ sufficiently near to $y$ since $X$ is irreducible. This is a contradiction. Hence $f\left(X_{D i}\right)=D$ for all $i$ so that $f_{D}$ is flat along $X_{D, 0}$.

Q.E.D.

LEMma 3. Let $f: X \rightarrow S$ be a proper flat morphism of complex spaces. Suppose that $S$ is reduced and irreducible. Suppose further that for some $o \in S, X_{o}$ is reduced and pure dimensional. Then $X$ also is reduced and pure dimensional.

Proof. Since $X_{o}$ is reduced, by Nakayama and the flatness of $f$ we infer readily that $X$ is reduced (cf. the proof of [9, Lemma 1.4]). To show the pure dimensionality it suffices to show that there is no irreducible component, say $X_{1}$, of $X$ such that if $q_{1}$ is the dimension of the general fiber of the induced map $X_{1} \rightarrow S$, then $q_{1}<q_{o}=\operatorname{dim} X_{o}$. Suppose that such an $X_{o}$ exists. Let $S_{k}(f)=\left\{x \in X ; \operatorname{codh}_{x} X_{f(x)} \leqq k\right\}$. Then $S_{k}(f)$ is an analytic subset of $X$ by [2]. Hence $S_{q_{1}}(f) \supseteq X_{1}, X_{1}$ being reduced, and so $\operatorname{dim} S_{q_{1}}(f)_{o} \geqq q_{1}$. Since $S_{q_{1}}(f)_{o}=\left\{x \in X_{o} ; \operatorname{codh}_{x} X_{o} \leqq q_{1}\right\}$, this implies that on $X_{o}$ there is a nonzero holomorphic function $\varphi$ with support of dimension $\leqq q_{1}\left(\right.$ cf. $[3$, p. 76 , Cor. $5.2 \mathrm{~d}) \rightarrow$ b)] applied to $\mathscr{F}=\mathcal{O}_{X}$ and $d=q_{1}$ ). This is a contradiction to the reducedness and pure dimensionality together of $X_{o}$. Hence $X$ is pure dimensional.

Q.E.D.

Proposition 3. Let $f: X \rightarrow S$ be a proper flat and surjective morphism of complex spaces. Suppose that $S$ is reduced and irreducible. Suppose further that for some $o \in S$ the fiber $X_{o}$ is reduced and irreducible. Then the general fiber of $f$ is irreducible.

Proof. By Lemma $3 X$ is reduced and pure dimensional. Apply Proposition 2 to $f$ and obtain a proper surjective morphism $\tilde{f}: \tilde{X} \rightarrow \tilde{S}$ and finite coverings $\alpha: \tilde{X} \rightarrow X$ and $\beta: \tilde{S} \rightarrow S$ with $\beta f=f \alpha$ satisfying the properties stated in the proposition. Let $\beta^{-1}(o)=\left\{\tilde{o}_{1}, \cdots, \tilde{o}_{m}\right\}$. Then it suffices to 
show that $\beta$ is locally biholomorphic at each $\tilde{o}_{k}$ and that $m=1$. In fact, then $\beta$ must be bimeromorphic and hence the irreducibility of the general fiber of $f$ follows from that of $\tilde{f}$ together with the surjectivity of $\alpha$. Now to prove the above assertion first we note that since $f$ is flat, $X$ is pure dimensional and $S$ is irreducible, every fiber of $f$ is pure dimensional of dimension $q=\operatorname{dim} X_{o}$, and, further, since $X_{o}$ is reduced and irreducible, every irreducible component of $X$ contains $X_{o}$. Combining this with 2) of Proposition 2 and the fact that $\left.\alpha\right|_{\tilde{X}_{\tilde{s}}}: \tilde{X}_{\tilde{s}} \rightarrow X_{\beta(\tilde{s})}$ is an embedding for each $\tilde{s} \in \tilde{S}$, we get that $\alpha$ induces the isomorphisms $\tilde{X}_{\tilde{o}_{k}} \cong X_{o}$ for all $k$. This then implies that $\beta$ is locally biholomorphic at each $\tilde{o}_{k}$, for otherwise $\tilde{X}_{\tilde{o}_{k}}$ is nonreduced at each of its points since so is $\tilde{S}$ at $\tilde{o}_{k}$ already. By Corollary above it also follows from $\tilde{X}_{\tilde{o}_{k}}=X_{o}$, that $\tilde{f}$ is flat in a neighborhood of $\tilde{X}_{\tilde{o}_{k}}$ for each $k$. Now we need the following result from [9, Cor. 3.3]; let $g: Y \rightarrow Z$ be a proper flat morphism of reduced complex spaces. Suppose that every fiber of $g$ has pure dimension $q$ which is independent of $z$. For any $z \in Z$ let $Y_{z, i}, i=1, \cdots, n=n(z)$, be the irreducible components of $Y_{z \text {, red }}$ and $m_{z, i}$ the multiplicities of $Y_{z}$ along $Y_{z \text {, red }}$ (cf. [9, 3.1]). Then for any continuous $(q, q)$-form $\chi$ on $X$ the function

$$
\lambda_{\chi}(z)=\sum_{i=1}^{n} m_{z, i} \int_{Y_{z, i}} \chi
$$

is a continuous function on $Z$. Using this we shall now show that $m=1$. Let $\omega$ be any Hermitian $(1,1)$-form on $X$ (cf. [9, Def. 1.2]) and set $\chi=$ $\omega \wedge \cdots \wedge \omega$ (q-times) and $\tilde{\chi}=\alpha^{*} \chi$. Then $\lambda_{\chi}(s)$ (resp. $\left.\lambda_{\tilde{\chi}}(\tilde{s})\right)$ are functions which are defined on $S$ (resp. $\tilde{S}$ ) and continuous in a neighborhood of $o$ (resp. $\beta^{-1}(o)$ ) by the result quoted above. Let $U$ (resp. $\tilde{U}_{k}$ ) be a neighborhood of $o$ (resp. $\tilde{o}_{k}$ ) such that $\beta$ induces isomorphisms $\beta_{k}: \tilde{U}_{k} \cong U$ for each $k$. For any $s \in U$ we write $\tilde{s}_{k}=\beta_{k}^{-1}(s)$. On the other hand, since $\alpha$ is bimeromorphic, there is a dense Zariski open subset $V$ of $U$ such that for each $s \in V, \tilde{X}_{\tilde{s}_{i}}, 1 \leqq i \leqq m$, are reduced and irreducible and $\alpha\left(\tilde{X}_{\tilde{s}_{k}}\right) \neq$ $\alpha\left(\tilde{X}_{\tilde{s}_{\ell}}\right)$ if $k \neq \ell$. Hence noting that $X_{s}=\bigcup_{k} \tilde{X}_{\tilde{s}_{k}}$ we have $\lambda_{x}(s)=\sum_{h=1}^{m} \lambda_{\tilde{z}}\left(\tilde{s}_{k}\right)$ for every $s \in V$. Now take a sequence $\left\{s^{(i)}\right\}$ of points of $V$ converging to $o$ in $U$. Then since $\lambda_{\chi}$ (resp. $\lambda_{\bar{\chi}}$ ) is continuous at $o$ (resp. $\tilde{o}_{k}$ ), we get that $\lambda_{\chi}(o)=\lim _{i} \lambda_{\chi}\left(s^{(i)}\right)=\lim _{i} \sum_{k=1}^{m} \lambda_{\tilde{\chi}}\left(\tilde{s}_{k}^{(i)}\right)=\sum_{k=1}^{m} \lambda_{\tilde{\chi}}\left(\tilde{o}_{k}\right)$. Since

$$
\lambda_{z}(o)=\int_{X_{o}} \chi=\int_{\tilde{X}_{\tilde{o}} k} \tilde{\psi}=\lambda_{\tilde{\chi}}\left(\tilde{o}_{k}\right) \text {, }
$$

this implies that $m=1$, for $\int_{X_{0}} \chi>0$.

Q.E.D. 


\section{$\S 4$. Moishezonness of $\pi_{A}$ in a special case}

(4.1) Let $f: X \rightarrow S$ be a proper morphism of complex spaces. Let $\beta_{X / S}: D_{X / S} \rightarrow S$ be the relative Douady space of $X$ over $S$ parametrizing analytic subspaces of $X$ contained in the fibers of $f$ (cf. [17], [9]). Let $\rho_{X / S}: Z_{X / S} \rightarrow D_{X / S}$ be the corresponding universal family, so that there is a natural embedding $Z_{X / S} \subseteq D_{X / S} \times_{S} X$ with $\rho_{X / S}$ induced by the natural projection $p_{1}: D_{X / S} \times_{S} X \rightarrow D_{X / S}$. We denote by $\pi_{X / S}$ the natural morphism $Z_{X / S} \rightarrow X$ induced by the projection $p_{2}: D_{X / S} \times_{S} X \rightarrow X$. Then $\pi_{X / S}$, restricted to each fiber of $\rho_{X / S}$, is an embedding. Let $\alpha: \tilde{S} \rightarrow S$ be a morphism of complex spaces with $\tilde{S}$ reduced and $Z \subseteq \tilde{S} \times{ }_{S} X$ a subspace. Let $\rho: Z \rightarrow \tilde{S}$ be the natural projection. If $\rho$ is flat, then we call $\rho$ a flat family of subspaces of $X$ over $S$ parametrized by $\tilde{S}$. In the general case, by Frisch [8] there is a dense Zariski open subset $W$ of $\tilde{S}$ such that $\rho_{W}: Z_{W} \rightarrow W$ is flat. (In what follows we use this result of Frisch without further reference.) Then there is a unique $S$-morphism $\tau: W \rightarrow D_{X / S}$ such that $\rho_{W}$ is isomorphic to the map induced from $\rho_{X / S}$ via $\tau$, where $W$ is over $S$ by $\left.\alpha\right|_{W}$. We call such a map $\tau$ simply the universal $S$-map associated to $\rho_{W}$.

Now we recall the following consequence of Hironaka's flattening theorem [14] which is of frequent use in the sequel.

Lemma 4. The universal S-map $\tau$ extends to a meromorphic S-map $\tau^{*}: \tilde{S} \rightarrow D_{X / S \text {, red. }}$ In particular if $\alpha$ is proper, then the closure of $\tau(W)$ in $D_{X / S, \text { red }}$ is an analytic subspace of $D_{X / S \text {, red }}$ which is proper over $S$.

Proof. See [9, Lemma 5.1].

(4.2) In the case of a projective morphism a special way of constructing $D_{X / S}$ is available by Grothendieck [12], [13]; what we need here from his construction is the following:

Lemma 5. Let $f: X \rightarrow S$ be a projective morphism and $\beta_{X / S}: D_{X / S} \rightarrow S$ be the relative Douady space of $X$ over $S$. Let $Q$ be any relatively compact open subset of $S$ and $A$ any connected component of $\beta_{X / S}^{-1}(Q)_{\mathrm{red}}$. Then the induced morphism $h: A \rightarrow Q$ is projective.

Proof. Let $Q^{\prime}$ be any relatively compact open subset of $S$ with $Q \subset$ $Q^{\prime}$. Let $\mathscr{L}$ be an $f_{Q^{\prime}}$-very ample invertible sheaf on $X$ such that $f_{*} \mathscr{L}$ is locally free on $Q^{\prime}$. So we have an $Q^{\prime}$-embedding $j: X_{Q^{\prime}} \rightarrow \boldsymbol{P}\left(f_{*} \mathscr{L}\right)_{Q^{\prime}}$ with $\mathscr{L} \cong j^{*} \mathcal{O}_{P}(1), \boldsymbol{P}=\boldsymbol{P}\left(f_{*} \mathscr{L}\right)$. Then replacing $S$ by $Q^{\prime}$ we may assume that $X=\boldsymbol{P}(\mathscr{E})$ for some locally free coherent analytic sheaf $\mathscr{E}$ on $S$. Now for 
$d \in D_{X / S}$ write $Z_{d}=Z_{X / S, d}$ and consider $Z_{d} \subseteq X_{\beta(d)} \cong C P^{r-1}$ by $\pi_{X / S}$, where $\beta=\beta_{X / S}$ and $r=\operatorname{rank} \mathscr{E}$. For every $d \in D_{X / S}$ define a polynomial $P_{d}=$ $P_{d}(n)$ in $n$ by $P_{d}=\sum_{i \geqq 0}(-1)^{i} H^{i}\left(Z_{d}, \mathcal{O}_{Z_{d}}(n)\right)$. Then $P_{a}$ is independent of $a \in A$ (cf. [3]) and we set $P_{A}=P_{a}$ for any $a \in A$. Set $\tilde{A}=\left\{d \in D_{X / S \text {, red }}\right.$; $\left.P_{d}=P_{A}\right\}$. Then $A$ is a connected component of $\tilde{A}_{Q}$. Hence it suffices to show that $\tilde{A}_{Q}$ is projective over $Q$. In fact, the proof of [13, IX, Théorème 1.1] (and [12, 221, §3]) shows that for each point $s \in S$ there exists a neighborhood $s \in U$ in $S$ and an integer $\nu_{0}=\nu_{0}(s)$ such that for all $\nu \geqq \nu_{0}$ the natural map $\beta^{*} f_{*} \mathcal{O}_{X}(\nu) \cong p_{1 *} p_{2}^{*} \mathcal{O}_{X}(\nu) \rightarrow \rho_{X / S *} \mathcal{O}_{Z_{X / S}}(\nu), \mathcal{O}_{Z_{X / S}}(\nu)=\pi_{X / S}^{*} \mathcal{O}_{X}(\nu)$, is surjective on $\tilde{A}_{U}$ and the corresponding morphism $\tilde{A}_{U} \rightarrow \operatorname{Grass}_{m}\left(f_{*} \mathcal{O}_{X}(\nu)\right)_{U}$ is a closed embedding over $U$, where $\operatorname{Grass}_{m}\left(f_{*} \mathcal{O}_{X}(\nu)\right)$ is the Grassmann variety of locally free quotients of $f_{*} \mathcal{O}_{X}(\nu)$ of rank $m$, where $m=m(\nu)=$ $\operatorname{rank}\left(\rho_{X / S *} \mathcal{O}_{Z_{X / S}}(\nu)\right)$ [13]. Hence for all sufficiently large $\nu, \tilde{A}_{Q}$ can be embedded in $\operatorname{Grass}_{m}\left(f_{*} \mathcal{O}_{X}(\nu)\right)_{Q}$ over $Q$ and hence is projective over $Q$. Q.E.D.

(4.3) Let $f: X \rightarrow S$ be a morphism of complex spaces. Let $\beta_{X / S}: D_{X / S}$ $\rightarrow S, \rho_{X / S}: Z_{X / S} \rightarrow D_{X / S}$ and $\pi_{X / S}: Z_{X / S} \rightarrow X$ be as in (4.1). For any locally closed analytic subspace $A$ of $D_{X / S \text {, red }}$ we shall denote by $\rho_{A}: Z_{A} \rightarrow A$ the restriction of $\rho_{X / S}$ to $Z_{A}=\rho_{X / S}^{-1}(A)$ and $\pi_{A}: Z_{A} \rightarrow X$ the $S$-morphism induced by $\pi_{X / S}$, where $Z_{A}$ is over $S$ by $\beta_{X / S} \rho_{A}$.

Proposition 4. Let $f: X \rightarrow S$ be a proper morphism of complex spaces and $Q$ a relatively compact open subset of $S$. Let $A$ be a reduced and irreducible analytic subspace of $\beta_{X / S}^{-1}(Q)$ which is proper over $Q$ and for which the general fiber of $\rho_{A}$ is reduced and irreducible. Then $\pi_{A}$ is Moishezon.

Proof. Changing the notation we set $S=Q, X=X_{Q}$ and $f=f_{Q}$ so that $A$ is an analytic subspace of $D_{X / S}$. (The original $X, S$ and $f$ do not appear explicitly in the following, so no confusion may arise). We first note that from our assumption it follows immediately that $Z_{A}$ is reduced and irreducible. Consider the $Z_{A}$-embedding $j: Z_{A} \times_{A} Z_{A} \subseteq Z_{A} \times_{X}\left(X \times_{S} X\right)$ defined by $j\left(z_{1}, z_{2}\right)=\left(z_{1}, \pi_{A}\left(z_{1}\right), \pi_{A}\left(z_{2}\right)\right.$ ) where $Z_{A} \times_{A} Z_{A}$ (resp. $X \times_{S} X$ ) is over $Z_{A}$ (resp. $X$ ) with respect to the projection to the first factor. Note that $j$ is in fact obtained by the composition $Z_{A} \times{ }_{A} Z_{A} \subseteq Z_{A} \times{ }_{A}\left(A \times{ }_{S} X\right)$ $\cong Z_{A} \times_{S} X \cong Z_{A} \times_{X}\left(X \times_{S} X\right)$ where the isomorphisms are all natural ones. On the other hand, let $\Delta=\Delta_{X / S}$ be the diagonal of $X \times_{S} X$ and $\mathscr{I}$ the sheaf of ideals of $\Delta$ in $X \times_{S} X$. Let $\Delta_{(n)}=\left(\Delta, \mathcal{O}_{X \times{ }_{S} X} / \mathscr{I}^{n+1}\right)$ be the $n$-th infinitesimal neighborhood of $\Delta$ in $X \times_{S} X$, and $\beta_{n}: \Delta_{(n)} \rightarrow X$ be induced by the projection $X \times{ }_{s} X \rightarrow X$ to the first factor. Then $\beta_{n}$ are finite, and 
hence projective, morphisms. Let $\delta_{n}: D_{(n)} \rightarrow X$ with $D_{(n)}=D_{\lrcorner_{(n)} / X}$ be the relative Douady spaces associated to $\beta_{n}$. Then for any connected component $D_{(n), k}$ of $D_{(n)}$ the induced morphism $D_{(n), k} \rightarrow X$ is projective by Lemma 5 since $f$ is proper.

Let $Y_{(n)}=\left(Z_{A} \times_{A} Z_{A}\right) \cap\left(Z_{A} \times_{X} \Delta_{(n)}\right) \subseteq Z_{A} \times_{X} \Delta_{(n)}$ and $\gamma_{n}: Y_{(n)} \rightarrow Z_{A}$ be the natural $S$-morphisms induced by the projections $Z_{A} \times_{X} \Delta_{(n)} \rightarrow Z_{A}$, where the intersection is taken in $Z_{A} \times_{X}\left(X \times_{S} X\right)$ considering $Z_{A} \times_{A} Z_{A}$ as a subspace of $Z_{A} \times_{X}\left(X \times_{S} X\right)$ via $j$. Then $\gamma_{n}$ are finite surjective morphisms, the fibers over $z \in Z_{A}$ being naturally identified with the subspace $B_{z, n}=$ $\pi_{A}\left(Z_{A, a}\right) \cap x_{(n)}$ of $x_{(n)}=\left(x, \mathcal{O}_{X} / m_{x}^{n+1}\right)$ where $a=\rho_{A}(z), x=\pi_{A}(z)$ and $m_{x}$ is the maximal ideal of $\mathcal{O}_{X}$ at $x$. Now for each $n$ there is a dense Zariski open subset $U_{n}$ of $Z_{A}$ such that $\gamma_{n, U_{n}}: Y_{(n), U_{n}} \rightarrow U_{n}$ is flat, so that it may be considered as a flat family of subspaces of $\Delta_{(n)}$ over $X$ parametrized by $U_{n}$. Let $\tau_{n}: U_{n} \rightarrow D_{(n)}$ be the universal $X$-map associated to $\gamma_{n, U_{n}}$ (cf. (4.1)). Then by Lemma $4 \tau_{n}$ extends to a meromorphic $X$-map $\tau_{n}^{*}: Z_{A} \rightarrow D_{(n)}$ and the closure $E_{n}$ of $\tau_{n}\left(U_{n}\right)$ in $D_{(n)}$ is analytic in $D_{(n)}$ and is proper over $S$ as well as $Z_{A}$.

Now we shall show that $(*) \tau_{n}^{*}$ are bimeromorphic X-maps onto its image for all sufficiently large $n$. Then since $\pi_{A}$ is proper and the images of $\tau_{n}^{*}$ are contained in some $D_{(n), k}, Z_{A}$ being irreducible, this would imply that $\pi_{A}: Z_{A} \rightarrow X$ is Moishezon by (1.7) 5), completing the proof of the proposition. To show $\left({ }^{*}\right)$ we first observe the following: (") If $z, z^{\prime} \in U_{m}$ $\cap U_{n}$ and if $m \leqq n$, then $\tau_{n}(z)=\tau_{n}\left(z^{\prime}\right)$ implies that $\tau_{m}(z)=\tau_{m}\left(z^{\prime}\right)$. In fact, for $z \in U_{n}, \tau_{n}(z)$ is the point of $D_{(n)}$ corresponding to the subspace $B_{z, n}$ of $x_{(n)}$ defined above, and that $B_{z, n}=B_{z^{\prime}, n}$ clearly implies that $B_{z, m}=B_{z^{\prime}, m}$. This shows ("). Now since $Z_{4}$ is irreducible, for each $n$ there exist an integer $d_{n} \geqq 0$ and a dense Zariski open subset $V_{n}$ of $U_{n}$ such that $\operatorname{dim}_{z} \tau_{n}^{-1} \tau_{n}(z)=d_{n}$ for all $z \in V_{n}$. Then we see that $d_{n} \leqq d_{m}$ for $m \leqq n$ by ("). Hence there are integers $n_{0}>0$ and $d \geqq 0$ such that $d_{n}=d$ for all $n \geqq n_{0}$.

Next we show that $d=0$. Let $W$ be a dense Zariski open subset of $A$ such that $Z_{A, a}$ is reduced and irreducible for all $a \in W$. Let $V=\bigcap_{n} V_{n}$. Then $V$ is everywhere dense in $Z_{A}$. Suppose now that $d>0$. Then there exist points $z, z^{\prime} \in V \cap \rho_{A}^{-1}(W), z \neq z^{\prime}$, such that $z^{\prime}$ belongs to an irreducible component $C$ of $\tau_{n_{0}}^{-1} \tau_{n_{0}}(z)$ containing $z$. (In particular $\pi_{A}(z)=\pi_{A}\left(z^{\prime}\right)$.) Then since both $Z_{A, \rho_{A}(z)}$ and $Z_{A, \rho_{A}\left(z^{\prime}\right)}$ are reduced and irreducible, there is an integer $n_{1} \geqq n_{0}$ such that $B_{z, n_{1}} \neq B_{z^{\prime}, n_{1}}$, or equivalently, $\tau_{n_{1}}(z) \neq \tau_{n_{1}}\left(z^{\prime}\right)$. Hence 
$\tau_{n_{1}, C}=\left.\tau_{n_{1}}\right|_{\cap \cap v_{n_{1}}}$ is nontrivial, i.e., the fibers of $\tau_{n_{1}, C}$ have dimension $<d=$ $\operatorname{dim} C$. Note here that $C \cap U_{n_{1}} \supseteq C \cap V \neq \emptyset$. On the other hand, by $\left.{ }^{\prime \prime}\right) \tau_{n_{1}, C}^{-1} \tau_{n_{1}}(z)$ is one of the irreducible components of $\tau_{n_{1}}^{-1} \tau_{n_{1}}(z)$ at $z$ and hence there is an irreducible component $C^{\prime} \subseteq C$ of $\tau_{n_{1}}^{-1} \tau_{n_{1}}(z)$ containing $z$, so that $\operatorname{dim}_{z^{\prime \prime}} \tau_{n_{1}}^{-1} \tau_{n_{1}}\left(z^{\prime \prime}\right)<d$ for some $z^{\prime \prime} \in C^{\prime}$. This implies that $d_{n_{1}}<d$ by the upper semi-continuity of the function $\operatorname{dim}_{z} \tau_{n_{1}}^{-1} \tau_{n_{1}}(z), z \in U_{n_{1}}$, which is a contradiction. Hence we get that $\tau_{n}$ is generically finite for $n \geqq n_{0}$.

Thus for each $n \geqq n_{0}$, there exist an integer $k_{n}>0$ and a dense Zariski open subset $Q_{n}$ of $E_{n}$ contained in $\tau_{n}\left(U_{n}\right)$ such that $\tau_{n, Q_{n}}: \tau_{n}^{-1}\left(Q_{n}\right)$ $\rightarrow Q_{n}$ is an unramified covering of degree $k_{n}$. Here one needs to recall that $\tau_{n}$ extends to a meromorphic $X$-map from $Z_{A}$ to $E_{n}$ which are both proper over $X$. Then again by ('), $k_{n} \leqq k_{m}$ if $n \geqq m$ so that $k_{n}=k$ for all $n \geqq n_{2}$ for some $k \geqq 1$ and $n_{2} \geqq n_{0}$. We show that $k=1$. Let $\tilde{Q}=$ $\bigcap_{n} \tau_{n}^{-1}(Q)$ which is everywhere dense in $Z_{A}$. For $\tilde{q} \in \tilde{Q}, \tau_{n}^{-1} \tau_{n}(\tilde{q})$, as a set, is independent of $n \geqq n_{2}$. Suppose that $k>1$. Then there are points $z$, $z^{\prime} \in \tilde{Q} \cap \rho_{A}^{-1}(W), z \neq z^{\prime}$, such that $\tau_{n_{2}}(z)=\tau_{n_{2}}\left(z^{\prime}\right)$. Then by the same argument as above we can find $n>n_{2}$ such that $\tau_{n}(z) \neq \tau_{n}\left(z^{\prime}\right)$, implying that $k_{n}<k$, since $z, z^{\prime} \in \tilde{Q}$. This contradicts our choice of $n_{2}$. Hence $k=1$, i.e., $\tau_{n}^{*}$ is $X$-bimeromorphic onto its image for all $n \geqq n_{2}$, and $\left(^{*}\right)$ is proved.

Q.E.D.

Remark. A meromorphic map $g: Y \rightarrow Y^{\prime}$ of reduced complex spaces is called generically light if there is a dense Zariski open subset $U \subseteq \Gamma$ such that $\operatorname{dim}_{r} q^{-1} q(\Gamma)=0$ for every $\gamma \in U$ where $\Gamma$ is the graph of $g$ and $q: \Gamma \rightarrow Y^{\prime}$ is the natural projection (cf. (1.1)). Then the above proof shows that even in the general case where $A$ may not be proper over $S$, there is a generically light meromorphic $X$-map $\lambda: Z_{A} \rightarrow B$ of complex spaces over $X$ with $B$ projective over $X$.

\section{$\S 5$. Reduction of the general case and proof of Theorem}

(5.1) We use the notation of (4.3).

Proposition 5. Let $f: X \rightarrow S$ be a morphism of complex spaces. Let $A$ be an irreducible component of $D_{X / S \text {, red }}$ which is proper over $S$ and for which $Z_{A}$ is reduced. Then there exist 1) reduced and irreducible analytic subspaces $B_{i}, i=1, \cdots, n$, of $D_{X / S}$ such that $B_{i}$ is proper over $S$ and the general fiber of $\rho_{B_{i}}: Z_{B_{i}} \rightarrow B_{i}$ is reduced and irreducible, 2) a reduced and irreducible analytic subspace $B$ of $\hat{B}=B_{1} \times{ }_{s} \cdots \times{ }_{S} B_{n}$ and 3) a generically 
surjective meromorphic $S$-map $h: B \rightarrow A$.

Proof. We write $Z=Z_{A}$ and $\rho=\rho_{A}$. Let $\tilde{\rho}: \tilde{Z} \rightarrow \tilde{A}, \alpha: \tilde{Z} \rightarrow Z$ and $\beta: \tilde{A} \rightarrow A$ be as in Proposition 2 applied to $f=\rho$. In particular $\tilde{Z} \subseteq \tilde{A}$ $\times_{A} Z$ with $\tilde{\rho}$ induced by the natural projection $\tilde{A} \times_{A} Z \rightarrow \tilde{A}$. Further since $\rho$ is flat, we may assume that $\beta$ is a finite covering. Moreover for each $s \in A, Z_{s}=\bigcup_{\tilde{s} \in \beta-1(s)} \alpha\left(\tilde{Z}_{\tilde{s}}\right)$ by 2) of the proposition. Then we apply Lemma 1 to $\beta$ and obtain a normal complex space $A^{\prime}$, a finite covering $\gamma: A^{\prime} \rightarrow A$ and an $A^{\prime}$-isomorphism $\lambda:\left(\tilde{A} \times{ }_{A} A^{\prime}\right)^{*} \cong E \times A^{\prime}$, where $\left(\tilde{A} \times{ }_{A} A^{\prime}\right)^{*}$ is the normalization of $\tilde{A} \times{ }_{A} A^{\prime}$ and $E$ is a finite set considered as a zero dimensional analytic space. Write $A^{*}=\left(\tilde{A} \times{ }_{A} A^{\prime}\right)^{*}$. Let $\rho^{*}: Z^{*} \rightarrow A^{*}$ be the pull-back of $\tilde{\rho}$ to $A^{*}$ with respect to the natural projection $A^{*} \rightarrow \tilde{A}$. Identifying $E$ with $\{1, \cdots, n\}, n=\# E$, in a certain fixed way and $A^{*}$ with $E \times A^{\prime}$ via $\lambda$, we write for each $i, Z_{\imath}^{*}=\rho^{*-1}\left(\{i\} \times A^{\prime}\right)$, and $\rho_{i}^{*}=\left.\rho^{*}\right|_{Z_{i}^{*}}: Z_{i}^{*}$ $\rightarrow\{i\} \times A^{\prime}=A^{\prime}$ and define $\pi_{i}^{*}: Z_{i}^{*} \rightarrow X$ to be the natural map. We thus get the following commutative diagram

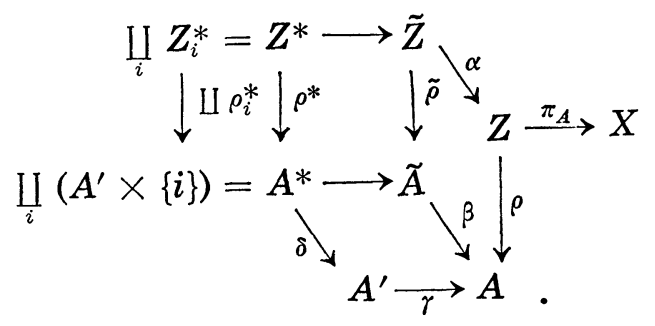

Let $U$ be any dense Zariski open subset of $A^{\prime}$ such that $\rho_{i, U}^{*}: Z_{i, U}^{*} \rightarrow U$ are flat for all $i$. Then by the definition of $\tilde{Z}$ we may consider $\rho_{i, U}^{*}$ naturally as a flat family of subspaces of $X$ over $S$ parametrized by $U$. Let $\tau_{i}: U$ $\rightarrow D_{X / S}$ be the universal $S$-map associated to $\rho_{i, U}^{*}$ and $\tau=\prod_{i} \tau_{i}: U \rightarrow$ $D_{X / S} \times{ }_{S} \cdots \times{ }_{S} D_{X / S}$ (n-times). Let $B$ (resp. $B_{i}$ ) be the closure of $\tau(U)$ (resp. $\left.\tau_{i}(U)\right)$ in $\hat{D}=D_{X / S} \times_{S} \cdots \times_{S} D_{X / S}$ (resp. $D_{X / S}$ ). Then by Lemma 4 (cf. also (1.1)) $B$ and $B_{i}$ are reduced analytic subspaces of $\hat{D}$ and $D_{X / S}$ respectively which are proper over $S$. They are irreducible since so is $U$, and we have $B \subseteq \hat{B}=B_{1} \times{ }_{S} \cdots \times{ }_{S} B_{n}$ and $\operatorname{dim} B \leqq \operatorname{dim} A$. Moreover since by 3 ) of Proposition 2 together with the definition of $\tau_{i}, Z_{X / S, d}$ is reduced and irreducible for each $d \in \tau_{i}(U)$ (after restricting $U$ if necessary), the general fiber of $\rho_{B_{i}}: Z_{B_{i}} \rightarrow B_{i}$ is reduced and irreducible. (For instance, since $\tau_{i}(U)$ is everywhere dense in $B_{i}$ it follows that $Z_{B_{i}}$ is reduced and irreducible. Then we can apply Proposition 3.)

Now let $\rho_{B}^{(i)}: Z_{B}^{(i)} \rightarrow B$ be the pull-back of $\rho_{B_{i}}$ with respect to the map 
$B \rightarrow B_{i}$ induced by the natural projection $\hat{B} \rightarrow B_{i}$. Take the union $\check{Z}_{B}=$ $\bigcup_{i} Z_{B}^{(i)}$ in $X \times_{S} B\left(\supseteq Z_{B}^{(i)}=Z_{B_{i}} \times_{B i} B\right)$. Let $\psi: \check{Z}_{B} \rightarrow B$ be the natural projection and take any dense Zariski open subset $V$ of $B$ such that $\psi_{V}: \psi^{-1}(V) \rightarrow V$ is flat. Let $\tau^{\prime}: V \rightarrow D_{X / S}$ be the universal $S$-map associated with $\psi_{V}$. Let $\bar{\pi}: \check{Z}_{B} \rightarrow X$ be the natural map induced by the projection $X \times{ }_{S} B \rightarrow X$. Then from the construction above we have in $X$ the equality $\bar{\pi}\left(\check{Z}_{B, b}\right)=\pi_{A}\left(Z_{A, \gamma(u)}\right)$ for each $b \in \tau(U)$ where $u \in U$ is any point with $\tau(u)$ $=b$. In fact by 2) of Proposition 2 we have $\pi_{A}\left(Z_{A, \gamma(u)}\right)=\bigcup_{\tilde{a} \in \beta-1_{\gamma}(u)} \approx\left(\tilde{Z}_{a}\right)$ $=\bigcup_{a^{*} \in \hat{o}^{-1}(u)} \pi^{*}\left(Z_{a^{*}}^{*}\right)=\bigcup_{i} \pi_{i}^{*}\left(Z_{i, u}^{*}\right)=\bar{\pi}\left(\check{Z}_{B, b}\right)$ in $X$, where $\tilde{\pi}=\pi_{A} \alpha$ and $\pi^{*}$ is the composite of $\tilde{\pi}$ and the natural map $Z^{*} \rightarrow \tilde{Z}$. This implies that $\left.\tau^{\prime} \tau\right|_{\tau^{-1}(V)}$ $=\left.j \gamma\right|_{\tau-1(V)}$ where $j: A \rightarrow D_{X / S}$ is the natural inclusion. In particular $\tau^{\prime}(V)$ contains $\gamma\left(\tau^{-1}(V)\right)$ and hence a nonempty Zariski open subset of $A$ since $\tau^{-1}(V) \neq \emptyset$. Thus the closure $\tau^{\prime}(V)^{-}$of $\tau^{\prime}(V)$ in $D_{X / S}$, which is an analytic subset of $D_{x / S}$ by Lemma 4 , contains $A$ so that $\operatorname{dim} B \geqq \operatorname{dim} A$. Combining with the opposite inequality noted above we have $\operatorname{dim} B=\operatorname{dim} A$, and thus $\tau^{\prime}(V)^{-}=A$. Hence $h=\tau^{\prime}$ is a generically surjective meromorphic $S$-map from $B$ onto $A$.

Q.E.D.

Remark. In fact the above $h$ is bimeromorphic as one shows readily.

(5.2) Theorem. Let $f: X \rightarrow S$ be a proper morphism of reduced complex spaces, and $Q$ a relatively compact open subset of $S$. Let $\beta: D_{X / S} \rightarrow S$ be the relative Douady space of $X$ over $S$. Suppose that $f \in \mathscr{C} / S$ (resp. is Moishezon). Then for any irreducible component $A$ of $\beta^{-1}(Q)_{\mathrm{red}}$ such that $Z_{A}$ is reduced, the induced morphism $\left.\beta\right|_{A}: A \rightarrow Q$ is proper and again belongs to $\mathscr{C} / S$ (resp. is Moishezon).

Proof. We shall write $f \in \mathscr{M} / S$ if $f$ is Moishezon. First we show that $A$ is proper over $S$. Since $f \in \mathscr{C} / S$ (resp. $\mathscr{M} / S$ ), there is a proper and locally Kähler (resp. locally projective) morphism $g: Y \rightarrow S$ of complex spaces and a surjective $S$-morphism $h: Y \rightarrow X$ (cf. (2.4) 2) and (1.5) 3)). Let $X^{\prime}=f^{-1}(Q), f^{\prime}=\left.f\right|_{X^{\prime}}: X^{\prime} \rightarrow Q, Y^{\prime}=g^{-1}(Q)$ and $g^{\prime}=\left.g\right|_{Y^{\prime}}: Y^{\prime} \rightarrow Q$. Then $g^{\prime}$ is Kähler (cf. (2.2) 1)). Hence by [9, Theorem 4.3] $g^{\prime}$ has property $B P$, i.e., every irreducible component of the relative Barlet space $B\left(Y^{\prime} / Q\right)$ (cf. [9]) is proper over $Q$. Then by [9, Prop. 4.8] $f^{\prime}$ also has property $B P$, which in turn implies that $f^{\prime}$ has property $\bar{D} P$, i.e., every irreducible component of $\bar{D}_{X^{\prime} / Q}$ is proper over $Q$, by [9, Prop. 3.4], where $\bar{D}_{X^{\prime} / Q}$ is the union of those irreducible components $D_{r}$ of $D_{X^{\prime} / Q \text {, red }}$ such that $Z_{r}=Z_{D_{r}}$ are reduced and pure dimensional. Then by [9, Lemma 3.5] and the remark 
following it (where $Z_{\alpha}$ and $D_{\alpha}$ should read $D_{\alpha}$ and $S$ respectively), this further implies that the given $A$ is proper over $S$ since $Z_{A}$ is reduced.

Now apply Proposition 5 to our $A$ and obtain $B \subseteq B_{1} \times_{Q} \cdots \times_{Q} B_{n}$ as in that proposition (with $S$ replaced by $Q$ ). In particular, $B_{i}$ are proper over $Q$, the general fiber of $\rho_{B i}: Z_{B_{i}} \rightarrow B_{i}$ is reduced and irreducible, and there is a generically surjective meromorphic $S$-map $B \rightarrow A$. The first two facts, together with Proposition 4, shows that $\pi_{B_{i}}: Z_{B_{i}} \rightarrow X^{\prime}$ is Moishezon, $1 \leqq i \leqq n$. Hence $f^{\prime} \rho_{B_{i}}: Z_{B_{i}} \rightarrow Q \in \mathscr{C} / Q$ (resp. $\mathscr{M} / Q$ ) by (2.4) 5 ) (resp. (1.5) 1)). Then by (2.4) 3) 4) and 7) (resp. (1.6) and (1.7) 5) 7)) the natural map $B_{i} \rightarrow Q$, and hence $B \rightarrow Q$ also, belong to $\mathscr{C} / Q$ (resp. are Moishezon). Finally by (2.4) 4) (resp. (1.6)) $\left.\beta\right|_{A}: A \rightarrow Q \in \mathscr{C} / Q$ (resp. $\mathscr{M} / Q$ ). Q.E.D.

Remark. Taking $S$ to be a point and then setting $S=Q$, we obtain the theorem stated in the introduction.

\section{Appendix}

We shall give a direct proof of Lemma 2 , in $\S 3$.

Let $D=\{t \in C ;|t|<1\}$ be the unit disc. For any complex space $Y$ and $y \in Y$ we denote by $S(Y, y)$ the set of morphisms $h: D \rightarrow Y$ with $h(0)$ $=y$. Let $f: X \rightarrow Y$ be a morphism of complex spaces and $y \in Y$. Then for any $h \in S(Y, y)$ we write $X_{h}$ for $X \times_{Y} D$ and $f_{h}$ (resp. $p_{h}$ ) for the natural projection $X_{h} \rightarrow D$ (resp. $X_{h} \rightarrow X$ ). Further for any coherent analytic sheaf $\mathscr{F}$ on $X$ we denote by $\mathscr{F}_{n}$ the $\mathcal{O}_{X_{h}}$-module $p_{h}^{*} \mathscr{F}$. Then Lemma 2 is a special case of the following:

Proposition. Let $f: X \rightarrow Y$ be a morphism of complex spaces and $\mathscr{F}$ a coherent analytic sheaf on $X$. Let $x \in X$ and $y=f(x)$. Suppose that $Y$ is reduced. Then the following conditions are equivalent: 1) $\mathscr{F}$ is f-flat at $x$. 2) For every $h \in S(Y, y), \mathscr{F}_{h}$ is $f_{h}$-flat at $x_{h}=(x, 0)$.

Proof. 2) is clearly a consequence of 1). So suppose that 2) is true. We use an analytic analogue of the technique due to Raynaud and Gruson $[19,2.1]$. Let $S(\mathscr{F})$ be the support of $\mathscr{F}$ considered as the analytic subspace of $X$ defined by the ideal sheaf of annihilators of $\mathscr{F}$. Then we proceed by induction on $n=\operatorname{dim}_{x}\left(X_{y} \cap S(\mathscr{F})\right.$ ). First replacing $X$ by $S(\mathscr{F})$ if necessary we may assume that $X=S(\mathscr{F})$, so that $n=\operatorname{dim}_{x} X_{y}$. Then there is a neighborhood $U$ of $x$ in $X$ and a commutative diagram of complex spaces 


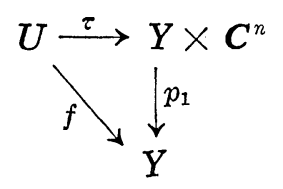

such that $\tau$ is finite at $x$ (cf. [7, 3.3]). Then since $\mathscr{F}_{x}$ and $\left(\tau_{*} \mathscr{F}\right)_{\tau(x)}$ are isomorphic as $\mathcal{O}_{Y, y}$-modules, we can replace $f$ and $\mathscr{F}$ by $p_{1}$ and $\tau_{*} \mathscr{F}$ respectively. Thus we may assume that $X=Y \times V$ with $Y$ Stein and $V$ a polydisc in $C^{n}$ containing the origin $0, x=(y, 0) \in Y \times C^{n}$ and $f: X \rightarrow Y$ is the natural projection.

Sublemma. $\mathscr{F}$ is locally free at some point of $X_{y}$.

Proof. For any $a \in X$ we set $d(a)=\operatorname{dim}_{C} \mathscr{F} \otimes_{O_{X}} \mathcal{O}_{X} / m_{a} \mathcal{O}_{X}$ where $m_{a}$ is the maximal ideal of $\mathcal{O}_{X}$ at $a$. Then $d(a)$ is upper semicontinuous with respect to the Zariski topology. In particular, if we set $d_{0}=\min \{d(a) ; a$ $\in X\}$, then the set $U_{0}=\left\{a \in A ; d(a)=d_{0}\right\}$ is Zariski open in $X$ and $\mathscr{F}$ is locally free on $U_{0}$. We may assume that $x \in \bar{U}_{0}$, the closure of $U_{0}$. Similarly if we put $d_{y 0}=\min \left\{d(a) ; a \in X_{y}\right\}$, then $U_{y 0}=\left\{a \in X_{y} ; d(a)=d_{y 0}\right\}$ is dense and Zariski open in $X_{y} \cong V$. We show that $d_{0}=d_{y 0}$. Take $h \in$ $S(Y, y)$ in such a way that $p_{h}^{-1}\left(U_{0}\right) \neq \emptyset$. By our assumption $\mathscr{F}_{h}$ is $f_{h}$-flat at $x_{h}$ and hence $f_{h}$-flat in some neighborhood $W$ of $x_{h}$. Since $D$ is smooth of dimension 1, this is equivalent to saying that $\mathscr{H}_{X_{h, 0}}^{0}\left(\mathscr{F}_{h}\right)=0$ on $W$. On the other hand, the latter implies that $\operatorname{dim} S_{n}\left(\mathscr{F}_{n}\right)<n$ (a special case of a theorem of Trautmann [3, p. 66]) where $S_{n}(\mathscr{F})=\left\{u \in X ; \operatorname{codh}_{u} \mathscr{F} \leqq n\right\}$, codh denoting the cohomological dimension. Hence for the general point $w \in X_{h, 0}, \operatorname{codh}_{w} \mathscr{F}_{h}=n+1$, i.e., $\mathscr{F}_{h}$ is locally free at $w$. Thus if $U_{h}$ is the maximal dense Zariski open subset of $X_{h}$ on which $\mathscr{F}_{h}$ is locally free, then $U_{h} \cap X_{y} \neq \emptyset$. Hence if $r$ is the rank of $\mathscr{F}_{h}$ on $U_{h}$, then taking any $a^{\prime} \in U_{h} \cap p_{h}^{-1}\left(U_{y 0}\right) \subseteq X_{h, 0}$ and $w^{\prime} \in U_{h} \cap p_{h}^{-1}\left(U_{0}\right)$ we have $d_{y 0}=d\left(p_{h}\left(a^{\prime}\right)\right)=$ $d_{h}\left(a^{\prime}\right)=r=d_{h}\left(w^{\prime}\right)=d_{0}$, where $d_{h}$ is defined for $\mathscr{F}_{h}$ in the same way as $d(a)$. Hence $U_{y 0} \subseteq U_{0}$ and $\mathscr{F}$ is locally free at each point of $U_{y 0} \subseteq X_{y}$.

Q.E.D.

By Sublemma there exists a $v \in V$ such that $\mathscr{F}$ is free of rank, say $r$, as an $\mathcal{O}_{X}$-module at $x^{\prime}=(y, v)$. We take $e_{1}, \cdots, e_{r} \in \Gamma(X, \mathscr{F})$ which give free generators of $\mathscr{F}$ at $x^{\prime}$. This is possible since $X$ is Stein. Let $\alpha: \mathcal{O}_{X}^{\oplus r}$ $\rightarrow \mathscr{F}$ be the map defined by $e_{i}$, and $\mathscr{K}$ (resp. $\mathscr{P}$ ) the kernel (resp. cokernel) of $\alpha$. Since $\alpha$ is isomorphic in a neighborhood of $x^{\prime}, \mathscr{K}=\mathscr{P}=0$ at $x^{\prime}$. In particular they are torsion $\mathcal{O}_{X}$-modules. Hence as a subsheaf of a free 
sheaf on the reduced space $X, \mathscr{K}$ must vanish identically on $X$. Thus we get an exact sequence

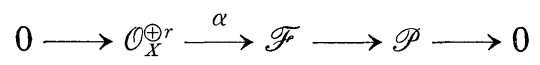

on $X$. Now we show that 2) is satisfied also for $\mathscr{P}$. For any $h \in S(Y, y)$, pulling back $(*)$ to $X_{h}$ we obtain the following exact sequence on $X$

$$
0 \longrightarrow \mathcal{O O}_{X}^{\oplus r} \stackrel{\alpha_{h}}{\longrightarrow} \mathscr{F}_{h} \longrightarrow \mathscr{P}_{h} \longrightarrow 0 \text {. }
$$

In fact, by the same reasoning as above, firstly $\alpha_{h}$ is isomorphic at $x_{h}^{\prime}=$ $\left(x^{\prime}, 0\right) \in X_{h}$ and then injective on the whole $X_{h}$ since $X_{h} \cong V \times D$ is reduced. Thus to show the flatness of $\mathscr{P}_{h}$ it is enough to show that for every integer $k \geqq 1$ the natural map $\alpha_{h}^{(k)}: \mathcal{O}_{X_{h}} / n^{k} \mathcal{O}_{X_{h}} \rightarrow \mathscr{F}_{h} / n^{k} \mathscr{F}_{n}$ induced by $\alpha_{h}$ is injective, where $n$ is the maximal ideal of $\mathcal{O}_{D, 0}$. In fact, by the flatness of $\mathscr{F}_{h}$ this implies that $\operatorname{Tor}_{1}^{R}(\mathscr{P}, R / a)=0$ for all ideals a of $R=$ $\mathcal{O}_{D, 0}$. Since $\alpha_{h}$ is isomorphic at $x_{h}^{\prime}$, so are $\alpha_{h}^{(k)}$ for all $k>0$. Thus if $\mathscr{K}_{k}$ $=\operatorname{Ker} \alpha_{h}^{(k)}, \mathscr{K}_{k}=0$ at $X_{h}^{\prime}$. Thus the support of $\mathscr{K}_{k}$ is a proper analytic subset of $X_{h, 0}$. Since $\mathscr{K}_{k} \subseteq \mathcal{O}_{X_{h}} / n^{k} \mathcal{O}_{X_{h}}$, it follows that $\mathscr{K}_{k}=0$. Hence $\mathscr{P}_{h}$ is $f_{h}$-flat, and 2) is verified for $\mathscr{P}$.

Now we finish the proof as follows. Recall that $\mathscr{P}_{x^{\prime}}=0$ so that $\operatorname{dim}_{x}\left(X_{y} \cap S(\mathscr{P})\right)<n$. If $n=0$, then $\mathscr{P}=0$ at $x$ so that $\mathscr{F}$ is free at $x$. So suppose that $n>0$. Then by induction and 2) for $\mathscr{P}, \mathscr{P}$ is $f$-flat at $x$. Then the flatness of $\mathscr{F}$ follows from $(*)$.

Q.E.D.

\section{REFERENCES}

[1] Artin, M., Algebraization of formal moduli, I, II, in Collected papers in Honor of K. Kodaira, Univ. of Tokyo Press and Princeton Univ. Press, (1969), 21-72 and Ann. of Math., 91 (1970), 88-135.

[ 2 ] Banica, C., Un théorème concernant les familles analytiques d'espaces complexes, Rev. Roumaine Math. Pures Appl., 18 (1973), 1515-1520.

[ 3 ] Banica, C. and Stanasila, O., Algebraic methods in the global theory of complex spaces, John Wiley \& Sons and Editura Academiei 1976.

[4] Barlet, D., Espace analytique reduit des cycles analytiques complexes de dimension finie, Seminaire F. Norguet, Lecture Notes in Math., No. 482, 1-158. Springer 1975.

[ 5 ] Campana, F., Algébricité et compacité dans l'espace des cycles d'un espace analytique complexe, Math. Ann., 251 (1980), 7-18.

[6] Douady, A., Le probléme de modules pour les sous-espaces analytique donné, Ann. Inst. Fourier, Grenoble, 16 (1966), 1-95.

[ 7 ] Fischer, G., Complex analytic geometry, Lecture Notes in Math., No. 538 Springer 1976.

[8] Frisch, J., Points de platitude d'un morphism d'espaces analytiques complexes, 
Inventiones math., 4 (1967), 118-138.

[9] Fujiki, A., Closedness of the Douady spaces of compact Kähler spaces, Publ. RIMS, Kyoto Univ., 14 (1978), 1-52.

[10] - On automorphism groups of compact Kähler manifolds, Inventiones math., 44 (1978), 225-258.

[11] - Deformations of uniruled manifolds, Publ. RIMS, Kyoto Univ., 17 (1981), $687-702$.

[12] Grothendieck, A., Fondements de la géométrie algébrique (Extraits du Seminaire Bourbaki 1957-1962), Paris 1962.

[13] —- Technique de construction en géométrie analytique, Seminaire Henri Cartan, $13^{\mathrm{e}}$ annee $(1960 / 61)$.

[14] Hironaka, H., Flattening theorem in complex-analytic geometry, Amer. J. Math., 97 (1975), 503-547.

[15] —- Bimeromorphic smoothing of a complex-analytic space, Math. Inst. Warwick Univ., England 1971.

[16] Hironaka, H., Leujeune-Jalabert, M. and Teissier, B., Platificateur local en géométrie analytique et applatissement local, Astérisque, 7-8 (1973), 441-463.

[17] Moishezon, B., Modifications of complex varieties and the Chow lemma, Lecture Notes in Math., No. 412, Springer 1974, 133-139.

[18] Pourcin, G., Théorème de Douady audessus de S, Ann. Sci. Norm. Sup. di Pisa, 23 (1969), 451-459.

[19] Raynaud, M. and Gruson, L., Critère de platitude de projectivité, Inventiones math., 13 (1971), 1-89.

[20] Rossi, H., Picard varieties of an isolated singular point, Rice Univ. Studies, 54 (1968), 63-73.

[21] Ueno, K., Classification theory of algebraic varieties and compact complex spaces, Lecture Notes in Math., No. 438, Springer 1975.

Kyoto University 